\title{
A case report on ACUM: a rare mullerian anomaly
}

\author{
Khushboo Malhotra*, Bindu Bajaj \\ Department of Obstetrics and Gynecology, VMMC and Safdarjung Hospital, New Delhi, India
}

Received: 09 January 2020

Accepted: 05 February 2020

\section{*Correspondence:}

Dr. Khushboo Malhotra,

E-mail: khushi_malh@yahoo.co.in

Copyright: $\odot$ the author(s), publisher and licensee Medip Academy. This is an open-access article distributed under the terms of the Creative Commons Attribution Non-Commercial License, which permits unrestricted non-commercial use, distribution, and reproduction in any medium, provided the original work is properly cited.

\begin{abstract}
Accessory and cavitated uterine mass (ACUM) is a rare, newly recognized mullerian anomaly. It is an accessory cavity lined by functional endometrium within an otherwise normal uterine cavity, in contrast to the other mullerian anomalies in which the uterus is malformed. It is often misdiagnosed as myoma, adenomyosis or adenomyoma. The entity needs expertise to diagnose as it is a rare but treatable cause of severe dysmenorrhea and chronic pelvic pain in young females with a wide range of differential diagnosis. A 30 years old female with history of one abortion 2 years back, came to infertility OPD with complaints of lower abdominal pain with severe dysmenorrhea, dyspareunia and inability to conceive since 2 years. Her menstrual cycle was regular with normal flow. USG pelvis showed subserosal fibroid of $4 \times 3 \mathrm{~cm}$ in fundus region of uterus. On HSG, bilateral fallopian tubes were patent. MRI-pelvis revealed normal uterus with thick walled cavitatory lesion of size $4.1 \times 3.6 \mathrm{~cm}$ with thick $\mathrm{T} 2 \mathrm{~W}$ hypointense wall similar to myometrium in continuation with right fundal wall of uterus containing blood products suggestive of ACUM. Both ovaries were normal, no obvious adnexal lesion/collection/free fluid noted in peritoneal cavity. Laparoscopy followed by laparotomy with excision of ACUM was performed. The MRI findings of an accessory cavitated uterine mass located below the attachment of round ligament with haemorrhagic contents, normal shaped uterus with normal bilateral tubes and ovaries should suggest the diagnosis of ACUM pre-operatively.
\end{abstract}

Keywords: Accessory cavitated uterine mass, Mullerian anomaly

\section{INTRODUCTION}

Mullerian duct anomalies consist of a set of structural malformations resulting from abnormal development of the Mullerian ducts. The prevalence of these anomalies' ranges from 0.001 to $10 \%$ in the general population and from 8 to $10 \%$ in women with adverse reproductive history. ${ }^{1}$ Septate uterus is the most common type. Accessory and cavitated uterine mass (ACUM) are a rare, newly recognized mullerian anomaly. It is an accessory cavity lined by functional endometrium within an otherwise normal uterine cavity, in contrast to the other mullerian anomalies in which the uterus is malformed. It is often misdiagnosed as myoma, adenomyosis or adenomyoma. The entity needs expertise to diagnose as it is a rare but treatable cause of severe dysmenorrhea and chronic pelvic pain in young females with a wide range of differential diagnosis.

\section{METHODS}

A 30 years old female with history of one abortion 2 years back, came to infertility OPD with complaints of lower abdominal pain with severe dysmenorrhea, dyspareunia and inability to conceive since 2 years. Her menstrual cycles were regular with normal flow. No history suggestive of pelvic inflammatory disease. General physical examination was normal and vitals were stable. Abdominal examination was normal. On perspeculum examination, cervix and vagina were healthy with no visible discharge. On per-vagina examination, uterus was retroverted, normal size, mass of around $4 \times 5$ 
$\mathrm{cm}$ was felt through right fornix, which was not felt separately from the uterus, left fornix was free, right fornix tenderness was present. On per-rectal examination, rectal mucosa was free, no nodularity felt.

USG pelvis showed subserosal fibroid of $4 \times 3 \mathrm{~cm}$ in fundus region of uterus. Bilateral ovaries were normal. No adnexal mass or free fluid in the pouch of douglas. Bilateral kidneys were normal. On HSG, bilateral fallopian tubes were patent. MRI-pelvis revealed normal uterus with thick walled cavitatory lesion of size $4.1 \times 3.6$ $\mathrm{cm}$ with thick $\mathrm{T} 2 \mathrm{~W}$ hypointense wall similar to myometrium in continuation with right fundal wall of uterus showing $\mathrm{T} 1 \mathrm{~W}$ and $\mathrm{T} 2 \mathrm{~W}$ hyperintense content suggestive of blood products making the diagnosis of ACUM. Both ovaries were normal, no obvious adnexal lesion/collection/free fluid noted in peritoneal cavity. Figure 1, 2, 3, 4 below shows the TAS, TVS, HSG and MRI images of our patient with ACUM, respectively.
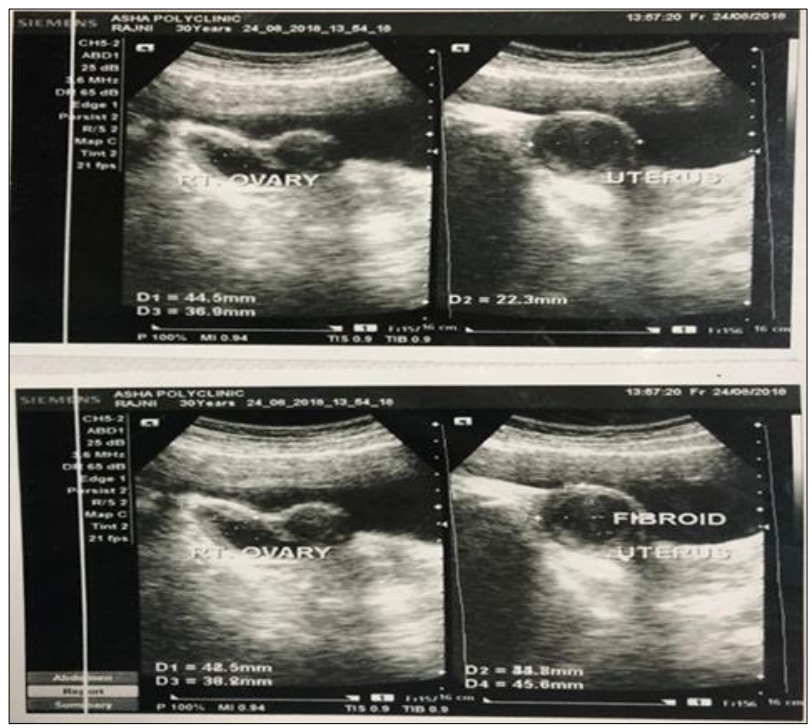

Figure 1: USG pelvis of subserosal fibroid of $4 \times 3 \mathrm{~cm}$ in fundus region of uterus.

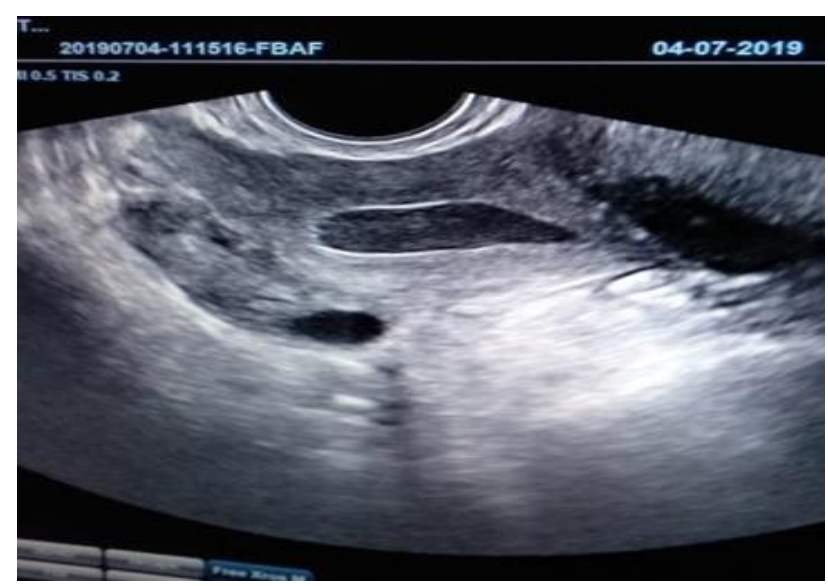

Figure 2: TVS of mass of size $4.5 \times 3.8 \mathrm{~cm}$ near the right cornua of uterus with old haemorrhagic contents.

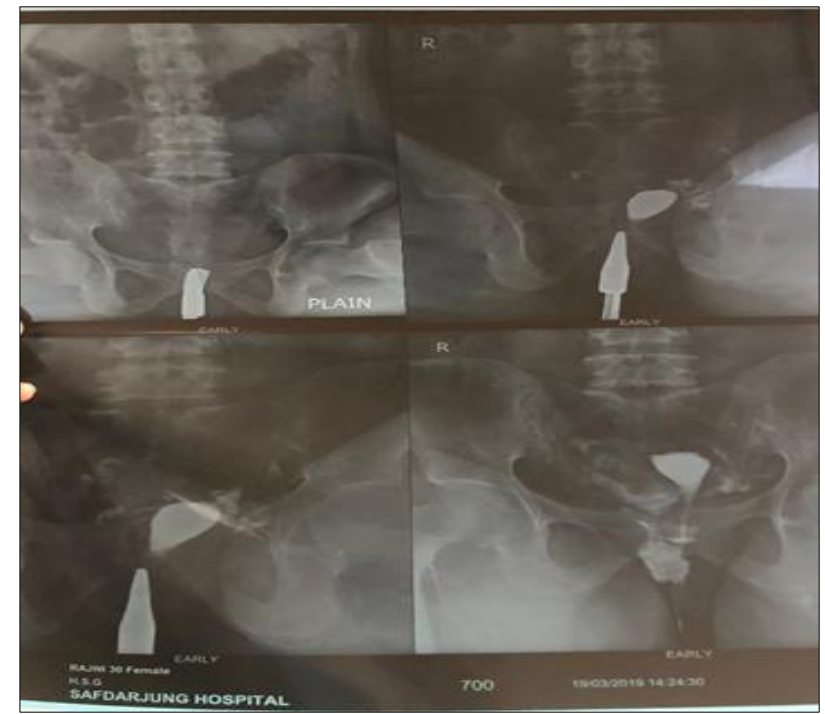

Figure 3: HSG of bilateral tubes patent.

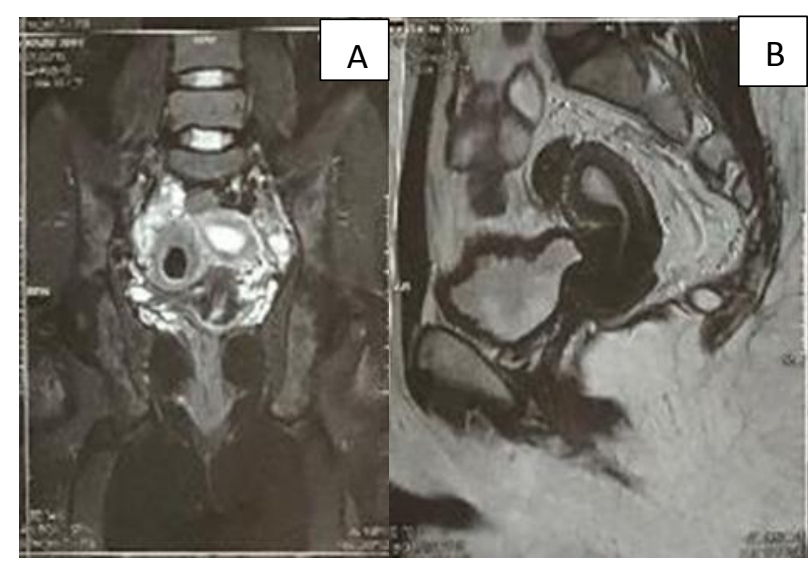

Figure 4: (A and B) MRI of ACUM - coronal view and sagittal view respectively.

On laparoscopy, undersurface of liver and gallbladder was normal. Bilateral fallopian tubes and ovaries were normal. Bilateral chromopertubation test was normal. A $4 \times 3 \mathrm{~cm}$ sized mass was observed arising near the right cornual end of the uterus making a differential diagnosis of cornual fibroid/cornual endometriotic cyst/ACUM. Figure 5 shows the laparoscopic image of this patient.

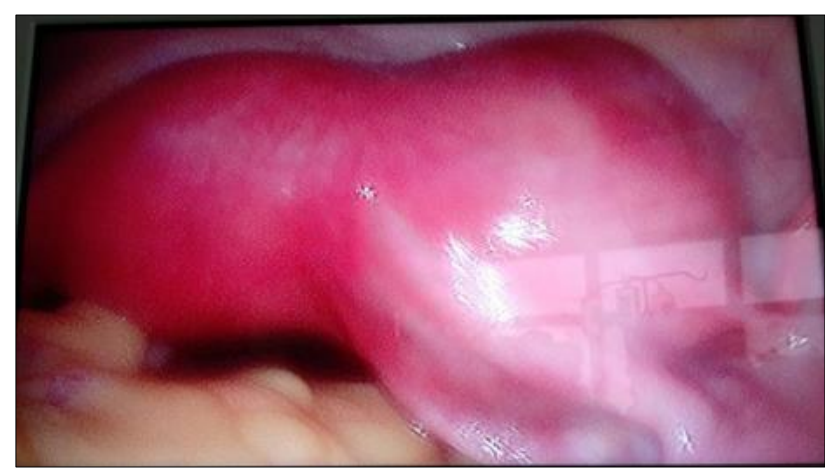

Figure 5: Laparoscopy showing ACUM. 
On laparotomy, uterus size was normal and a mass of size $4 \times 3 \mathrm{~cm}$ was observed near the right cornua of the uterus. Transverse incision of $1 \times 1 \mathrm{~cm}$ was given over the mass and around $15 \mathrm{ml}$ of chocolate coloured fluid was drained from the cavitated lesion. Mass was excised by doing circumferential subcapsular/subserosal plane dissection and sent for histopathological examination. Since mass could be easily excised without opening the uterine cavity with bilateral normal attachment of fallopian tubes and round ligament to the original uterine cavity, possibility of uterine anomaly (bicornuate uterus) with hematometra was ruled out and thus the diagnosis of ACUM was confirmed. Uterus wall was stitched in layers by 3-0 vicryl. Baseball sutures were taken over serosa. Uterine cavity was not opened. Haemostasis was achieved. Blood loss was average. Patient stood the procedure well. Figure $6,7,8,9,10,11$ below shows the intra-op findings of this patient.

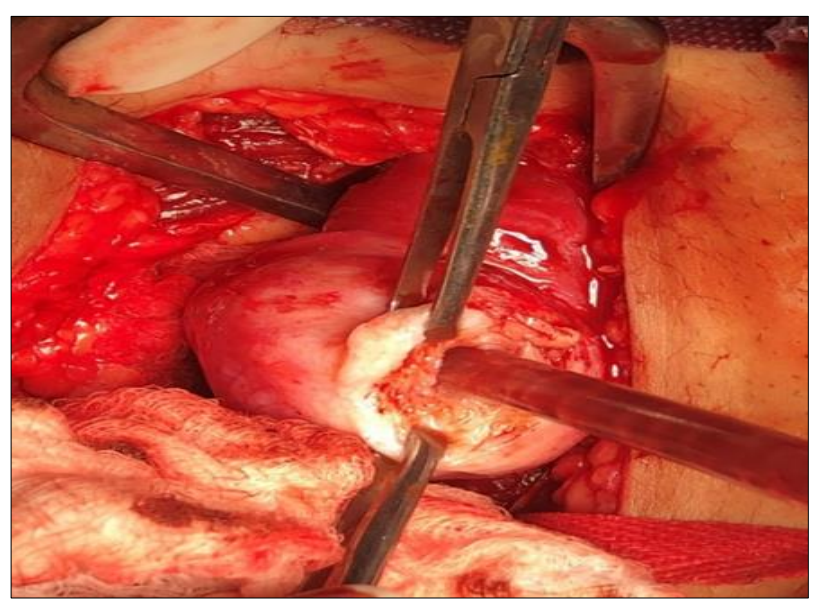

Figure 6: Drainage of chocolate coloured fluid through the incision made on the ACUM.

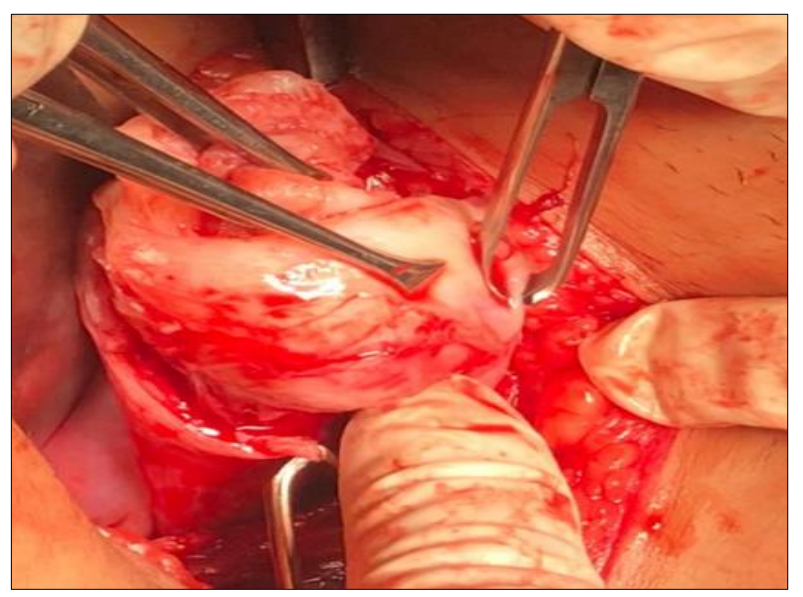

Figure 7: Circumferential subcapsular/subserosal plane dissection.

On cutting the specimen, a cavity was identified. The wall thickness was variable $0.5 \mathrm{~cm}$ to $1 \mathrm{~cm}$. Inner surface was irregular and hemorrhagic. Histopathological examination showed accessory uterine cavity with a lining epithelium with large collection of hemosiderophages. The surrounding myometrial wall was unremarkable. Thus, histopathological features were compatible with clinical diagnosis.

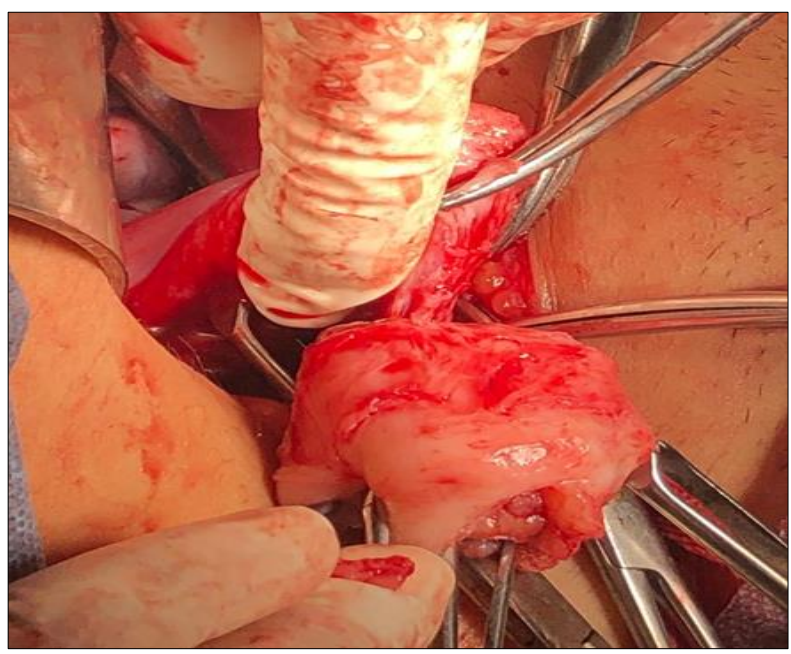

Figure 8: Excision of ACUM.

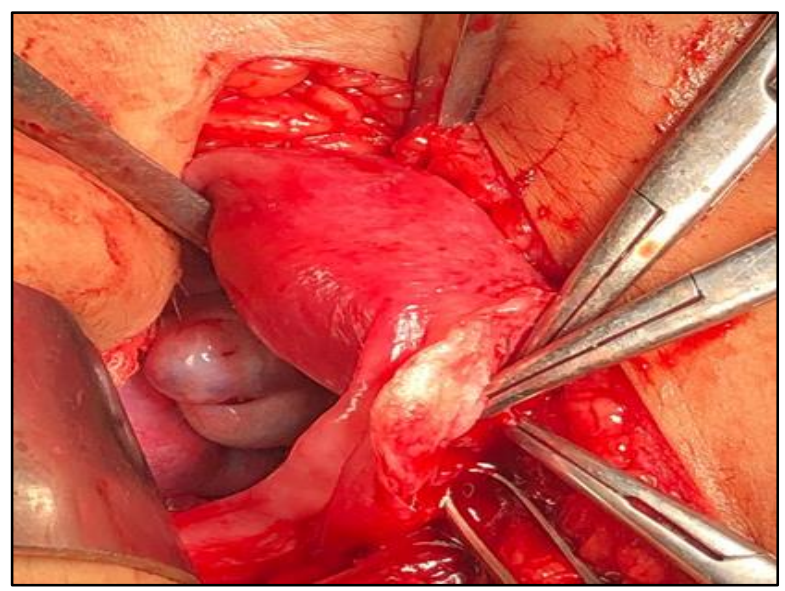

Figure 9: Uterine cavity (unopened) after the excision of ACUM.

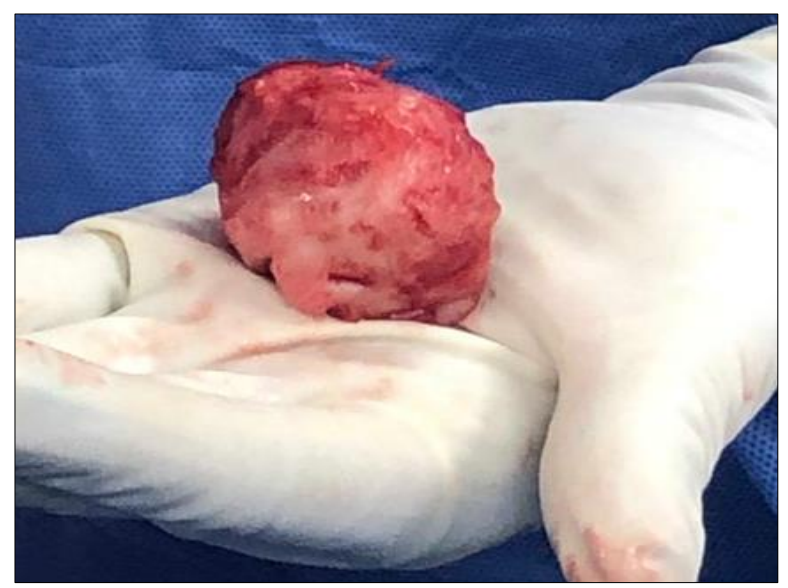

Figure 10: ACUM sent for histopathological examination. 


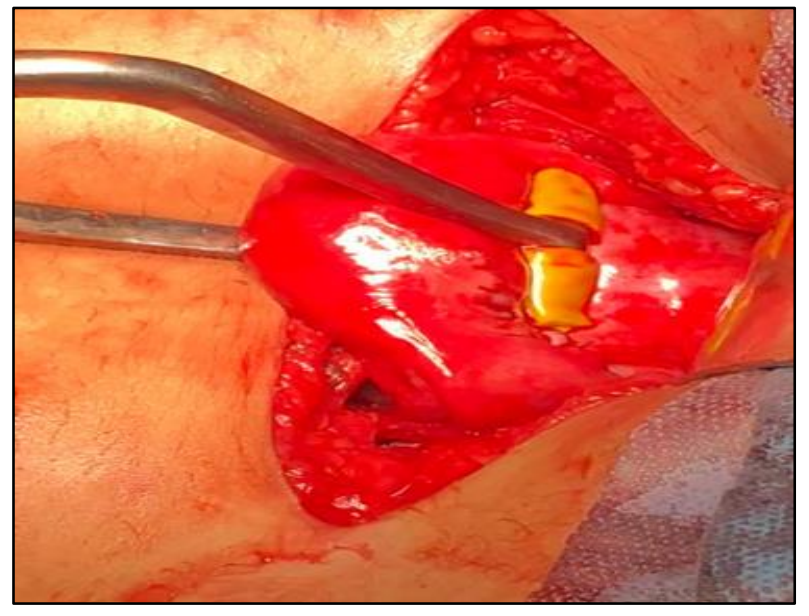

Figure 11: Uterus wall stitched back by baseball sutures.

\section{DISCUSSION}

Uterus develops from embryonic fusion of two mullerian ducts. Different classifications of mullerian anomalies have been given by ESHRE and ASRM. ASRM provides seven classes of mullerian anomalies. ${ }^{2}$

ACUM is a non-communicating uterus-like mass (ULM) arising in the uterus itself with uterine cavity and myometrium normal. It resembles uterus on microscopy. It characteristically presents at a younger age, usually < 30 years, with severe dysmenorrhea and chronic pelvic pain due to distention of the cavity caused by repeated bleeding. Various authors have previously described such masses with different names such as juvenile cystic adenomyoma (JCA), accessory cavitated masses, cavitated adenomyoma etc., essentially representing the same entity now termed as ACUM. ${ }^{3}$

\section{There are three theories of development}

1. Congenital anomaly theory

2. Heterotopias theory

3. Metaplasia theory. ${ }^{4}$

Most of the authors accept ACUM as a congenital anomaly. The proposed mechanism says that the accessory mass could be caused by duplication of ductal Mullerian tissue in the critical area at the level of attachment of round ligament, possibly related to gubernaculum dysfunction. ${ }^{5}$ Association with genitourinary and gastrointestinal anomalies have been described. ${ }^{4}$

The first case of ULM was reported by Cozzutto in 1981 . Around 36 cases of ACUM have been reported in literature with various terminologies, of which 22 have been reported after 2010 probably due to increased awareness. Beginning from 2010, the greatest number of cases $(n=9)$ has been reported by Takeuchi et al, although they limited the inclusion criteria to women under 30 years of age. Kriplani et al, reported four cases in 2011, and one case each was reported by Akar et al. in 2010 and Chun et al. in 2011. In 2012, Jain reported two cases of JCA, simulating Mullerian anomalies. In 2013, another case with its laparoscopic management has been reported by Bedaiwy et al. Till now, around 31 cases of ULM arising outside uterus have been described. The most common extrauterine site is ovary; however, such masses have been seen in broad ligament, small bowel, mesentery, appendix, colon, conus medullaris, and uterosacral ligament. ${ }^{3}$

\section{The criteria for diagnosing ACUM are}

1. An isolated accessory cavitated mass usually located under round ligament

2. Normal uterus, fallopian tubes, and ovaries

3. A surgical case with excised mass and pathological examination

4. An accessory cavity lined by endometrial epithelium with glands and stroma

5. Chocolate brown coloured fluid contents

6. No adenomyosis in the uterus (if resected), although there could be tiny foci of adenomyosis in the myometrium of the accessory cavity due to increased intra-cystic pressure. ${ }^{3}$

USG is the initial imaging modality that can identify them as solid isoechoic to predominantly cystic masses resembling endometrioma arising within the uterus, visualized separately from the ovaries. HSG is important in ruling out any mullerian anomaly. MRI is the imaging modality of choice as it is non-invasive and, hence, preferred over HSG in young unmarried females. It clearly shows the pelvic anatomy; cavitated mass with hemorrhagic contents; and the uterus, myometrium, and endo-myometrial interface. In our case, visualization of normal size and shape of the uterus and both cornua ruled out Mullerian anomalies. As bilateral ovaries were separate and appeared normal, ovarian tumours were excluded. Cystic degeneration in adenomyoma and fibroid will not show T2-hyperintense endometrial lining and hemorrhagic contents.

Regarding therapeutic management, most recent publications have included laparoscopic excision of the mass. Most of the cases were misdiagnosed preoperatively as other mullerian anomalies, cystic degeneration in adenomyoma and leiomyoma, and broad ligament fibroids. Awareness and adequate knowledge of the entity can help us make an accurate pre-operative diagnosis of ACUM.

\section{CONCLUSION}

ACUM, a rare Mullerian anomaly related to dysfunction of gubernaculum, is a treatable cause of severe dysmenorrhea in young females. MRI is highly accurate in making the diagnosis. The MRI findings of an accessory cavitated ULM located below the attachment of round ligament usually with haemorrhagic contents, an 
otherwise normal-shaped uterus with bilateral normal tubes and ovaries, without any evidence of adenomyosis should suggest the diagnosis of ACUM pre-operatively.

\section{ACKNOWLEDGMENTS}

Authors would like to thankful to Dr. Bindu Bajaj, senior specialist and associate professor in the department of obstetrics and gynecology in Safdarjung Hospital, for her guidance, constant supervision, continuous encouragement and helping in carrying out various procedures involved in this case. Authors are thankful to institute, VMMC and Safdarjung Hospital, for giving the opportunity to conduct the procedures involved in this case and proving the required equipment for the same.

Funding: No funding sources Conflict of interest: None declared Ethical approval: Not required

\section{REFERENCES}

1. Nath J, Pathak N. A study of mullerian anomalies in a tertiary care teaching hospital of North India. IJSR 2015;4(1):1920-2.
2. The American Fertility Society classifications of adnexal adhesions, distal tubal occlusion, tubal occlusion secondary to tubal ligation, tubal pregnancies, müllerian anomalies and intrauterine adhesions. Fertil Steril. 1988;49:944-55.

3. Jain N, Verma R. Imaging diagnosis of accessory and cavitated uterine mass, a rare mullerian anomaly. Indian J Radiol Imaging. 2014;24:178-81.

4. Na KY, Kim GY, Won KY, Kim HS, Kim SW, Lee $\mathrm{CH}$, et al. Extrapelvic uterus-like masses presenting as colonic submucosal tumor: a case study and review of literature. Korean J Pathol. 2013;47:177-81.

5. Acién P, Sánchez del Campo F, Mayol MJ, Acién M. The female gubernaculum: Role in the embryology and development of the genital tract and in the possible genesis of malformations. Eur $\mathrm{J}$ Obstet Gynecol Reprod Biol. 2011;159:426-32.

Cite this article as: Malhotra K, Bajaj B. A case report on acum: a rare mullerian anomaly. Int $\mathbf{J}$ Reprod Contracept Obstet Gynecol 2020;9:1310-4. 